\title{
El programa Sirchal: una aportación a la revitalización de centros históricos en América Latina y el Caribe
}

Léo Orellana *

En las últimas décadas del siglo xx, Europa y América Latina convergen en su preocupación por un tema dominante: la ciudad. Es éste un período de crecimiento demográfico acelerado, industrialización y urbanización, que modifica radicalmente la fisonomía urbana y socio-política. Esta inevitable mutación se acompaña de desequilibrios y cambios que dificultan el reconocerse en una ciudad que ha perdido su forma, su identidad y su definición.

En Europa, la ciudad es durante largo tiempo sinónimo de densidad y jerarquización del espacio. Este fenómeno, producto del hecho mismo de habitarla, necesita tiempo para manifestarse. Puede afirmarse que los centros históricos, comparados con las zonas periféricas recientes, se enriquecen a partir de las múltiples huellas que deja el tiempo, lo que favorece su densidad y su lectura. La ciudad es memoria, es una entidad viva, un flujo continuo que necesita tiempo para crear vínculos.

En nuestra época, la velocidad y los flujos acrecientan la movilidad de las formas y expresiones urbanas, por lo que el acto de construir se debe analizar a la luz de esa nueva realidad. Han quedado atrás los días en que se elogiaba la cultura de la perennidad. La arquitectura debe redefinirse: es el arte de transformar, de crear sobre lo existente.

El acto de construir debe acabar con el romanticismo de lo definitivo, lo único y lo monumental. Las intervenciones de los arquitectos tienen que ser ciudadanas y cotidianas; deben garantizar la calidad de vida y defender los valores humanos. Actualmente se revisan en Francia las acciones sobre lo cons- truido teniendo en cuenta estas exigencias y se trata de adaptar la arquitectura a las condiciones de la ciudad contemporánea, reevaluando los límites de la intervención de los arquitectos en el creciente mercado de la rehabilitación, la renovación y la restauración, sin olvidar que para la ciudad el tiempo es siempre más largo que para los hombres. Se redefine el concepto mismo de arquitectura: es el arte de transformar, de crear sobre lo ya existente.

«El desarrollo urbano debe apoyarse en los cimientos de la memoria» ${ }^{1}$ : en efecto, las ciudades deben envejecer, y al mismo tiempo renovarse, lo que redunda en la oposición espacial entre centros sobreprotegidos y zonas periféricas abandonadas a una perpetua renovación.

El patrimonio arquitectónico no se limita a los períodos más remotos, se extiende a los siglos $\mathrm{XIX}$ y $\mathrm{XX}$, tan presentes en las ciudades latinoamericanas; no está formado únicamente por monumentos prestigiosos, incluye también la trama urbana ordinaria y los espacios públicos, que constituyen el entorno de los habitantes de la ciudad y su capital social.

En Francia, los problemas vinculados con la gestión urbana con frecuencia son producto de falta de prospectiva, reflexión previa o coordinación, por lo que se ha aprovechado esa experiencia para desarrollar instrumentos que favorezcan la acción concertada. Cabe señalar aquí que el proceso de rehabilitación implica voluntad política, herramientas administrativas adecuadas y un marco jurídico adaptado a la problemática actual, lo suficientemente flexible como para permitir la evolución de la ciudad. 
La búsqueda del equilibrio inspirado en las cartas de Venecia y Granada rechaza la ruptura con el pasado y el plagio, actualizando así el espacio, sin romper con la identidad urbana. La arquitectura es evidentemente un acto político que no puede desligarse de la evolución del proceso democrático. En Francia son pocos los arquitectos comprometidos con la vida política de sus ciudades; es mucho lo que se puede aprender de América Latina, donde varias ciudades tienen o han tenido un alcaldearquitecto. En la actualidad la democracia se manifiesta en el terreno cultural y social; privilegiando el acceso a la educación, la cultura y la calidad de vida. Al convertirse en un acto cívico ordinario, la creación arquitectónica y los arquitectos pueden redefinir su campo de acción.

Las decisiones sobre los centros históricos deben ser concertadas. Una gestión compartimentada que no tenga en cuenta las zonas periféricas es contraproducente: por una parte, los centros históricos son presa fácil del proceso de «museificación», de la especulación funcional y de la degradación; por otra, las zonas periféricas recientes se limitạn esencialmente a ofrecer vivienda y transporte a los ciudadanos, sin que se lleguen a crear los vínculos tradicionales. En Francia, las políticas del Ministerio de Cultura, inspiradas en las clasificaciones del siglo XIX, contribuyeron en el pasado a promover esas divisiones compartimentadas. El actual proyecto interministerial para la ciudad favorece una acción global, basada en la reflexión y la gestión interdisciplinar. Sólo se logrará revitalizar la ciudad si se promueve una acción general coherente, que incluya la dimensión cultural, social y económica, y al mismo tiempo restituya el valor del espacio público, ya que la crisis de los centros históricos se debe en gran medida a la desaparición de tales espacios, que son lugares privilegiados de intercambio y socialización.

El contenido cultural y la temporalidad de nuestras ciudades llevan a reevaluar el papel que desempeñan el patrimonio, la cultura, la historia y la creación contemporánea. No se pueden concebir dos ciudades antagónicas y separadas: una histórica y central, con aires de museo, y otra reciente y periférica, carente de memoria. La ciudad es un conjunto y la revitalización de los centros históricos - que debe ser parte de una visión global, de un verdadero proyecto de desarrollo integrado a una reflexión sobre la temporalidad de la ciudad en general, y de cada ciudad en particular-, corresponde a la necesidad de contar con una ciudad heterogénea, viva y humana, en la que se observe una tendencia a la disminución de la segregación.

Durante mucho tiempo la práctica francesa estuvo dominada por la oposición entre creación y patrimonio, así como por intervenciones aisladas en la ciudad, pero esa experiencia ha demostrado sus límites. Hoy en día, se prefiere la interacción de memoria y proyecto, teniendo siempre en cuenta la totalidad del espacio construido, sin distinción de origen temporal. Hace algunos años, el Ministerio de Cultura de Francia decidió reunir la arquitectura y el patrimonio en una misma dirección: fue el primer paso para poner en práctica el concepto de memoria y proyecto; el cual demostró la necesidad de una reflexión permanente sobre este tema.

La ciudad contemporánea no es una simple superposición de elementos heterogéneos, con centros «históricos» inamovibles que desempeñan una función determinante, pero insuficiente, de identidad; es un establecimiento humano en constante evolución. Una improbable interrupción de este proceso y la separación de dos entidades incompatibles: una falsa verdadera ciudad «histórica» y una verdadera falsa ciudad «nueva» pueden provocar la agonía de una ciudad.

En Francia, este enriquecedor proceso de reflexión sobre la fisonomía urbana y el tejido construido se materializa en incontables proyectos que no sólo han logrado rearticular las identidades urbanas y restablecer la continuidad temporal, espacial y social de la ciudad, sino también contribuir a que ésta se reinvente en un proceso endógeno y en la adquisición de un considerable acervo en la materia. En efecto, a nivel internacional se reconoce a la experiencia francesa su aptitud para analizar la especificidad de un sitio, su historia, su entorno cultural y para proponer medidas adecuadas a cada caso, destinadas a valorizar su carácter patrimonial, asegurando al mismo tiempo su adaptación a las necesidades y u'sos contemporáneos.

Cuando la Dirección de Arquitectura y Patrimonio (DAPA) del Ministerio de Cultura y Comunicación de Francia -con el apoyo del Banco Interamericano de Desarrollo (BID), la UNESCO y la Unión Internacional de Arquitectos (UIA), así como numerosos protagonistas locales y representantes del sector privado- pone en marcha el programa Sirchal de revitalización de centros históricos de las ciudades de América Latina y el Caribe, coinciden varios factores: la UNESCO trabaja activamente en el tema del patrimonio cultural; la lista de ciudades latinoamericanas que forman parte del patrimonio mundial de la humanidad aumenta y prevalece en el mundo una situación económica neoliberal que genera privatizaciones del patrimonio económico, que a veces utilizan como vitrina la riqueza arquitectónica. En América Latina, cada país, cada ciudad, empieza a buscar, dentro de ese contexto «neoliberal», su propia identidad como contrapeso a la mundialización, agregándole un componente de desarrollo social y cultural. Al mismo tiempo, en Europa se desarrolla y pone en práctica el concepto de industrias culturales ${ }^{2}$.

El Banco Interamericano de Desarrollo, a través de su presidente, Enrique Iglesias, manifiesta el decidido apoyo de esta institución a la educación y la cultura, que constituyen inversiones de alta rentabilidad económica y social, tanto para sus beneficiarios como para la sociedad en general. Considera que los proyectos de infraestructura física orientados a recuperar el patrimonio cultural constituyen un nuevo producto que rea- 
firma la identidad, genera empleos en el sector turístico y proporciona creativas soluciones habitacionales ${ }^{3}$, pero observa que un desarrollo desenfrenado del sector puede suponer riesgos y tener efectos nocivos, contrarios a los beneficios esperados.

Durante varias décadas los especialistas en la conservación del patrimonio arquitectónico han restaurado, preservado y rehabilitado de manera idéntica al monumento original; pero en ese momento, más allá de la rehabilitación patrimonial, lo que se busca con la revitalización es dar nueva vida a los centros históricos en el contexto de la ciudad contemporánea. En esas condiciones se inicia el programa Sirchal. Es un programa que sostiene que no existen modelos, sólo referencias, y que los actores locales deben definir, iniciar y desarrollar proyectos sostenibles para los cuales la financiación debe comprender una importante contraparte local, que incluya tanto al sector público como al sector privado. Asimismo, permite confrontar, analizar y compartir las experiencias latinoamericanas y europeas.

Los objetivos iniciales del programa Sirchal son los siguientes:

- Reunir a los responsables de proyectos de rehabilitación de centros históricos de América Latina y el Caribe y crear una red —un espacio de diálogo-, que permita, más allá de las fronteras, un intercambio fecundo de experiencias. La red está destinada a los responsables de proyectos de rehabilitación, dado que existen otras redes utilizadas por instituciones y autoridades políticas.

- Analizar, a través de la información recabada por medio de la red, las diferentes experiencias de cooperación bilateral y multilateral, así como con los organismos internacionales.

El programa Sirchal se estructura alrededor de varios ejes de intervención: seminarios internacionales, talleres sobre un tema específico con participación de expertos nacionales e internacionales; presentaciones de proyectos de ciudades de América Latina, un sitio Internet y diversas publicaciones.

En el primer seminario internacional Sirchal realizado en París en mayo de 1998 se constituye una red de responsables y directores de proyecto de ciudades latinoamericanas y del Caribe, cuyo propósito es encontrar y definir denominadores comunes, conservando al mismo tiempo las particularidades de cada proyecto. También se debaten los siguientes temas: necesidad de buscar la concertación entre actores políticos, técnicos y financieros para mejorar la gestión de los recursos destinados a los proyectos de rehabilitación de centros históricos, participación de la sociedad civil en el proceso de toma de decisiones y valorización de aquellas experiencias de revitalización de centros históricos que estén más avanzadas y puedan convertirse en una referencia, creando así un proceso dinámico.

En el segundo seminario internacional efectuado en Quito, Ecuador, en noviembre del mismo año, se llega a las siguientes conclusiones: la creciente participación del sector privado constituye uno de los elementos motores de la dinámica de rehabilitación de centros históricos en América Latina; en el contexto del desarrollo urbano, la integración de los diferentes actores se facilita definiendo, junto con los entes municipales y/o administrativos de cada ciudad, nuevas herramientas y mecanismos de acción y, por último, para costear los programas de vivienda social en los centros históricos, que permiten retener a la población residente, es necesario desarrollar nuevos sistemas de financiación o adaptar los ya existentes para que integren diferentes fuentes (públicas, privadas, municipales o internacionales).

En el tercer seminario, organizado en Santiago y Valparaíso, Chile, en mayo de 1999, se analizan diferentes legislaciones existentes - tanto en América Latina y el Caribe, como en Francia-, así como las nuevas herramientas que permiten integrar diversos actores públicos o privados en los proyectos, los criterios de intervención (arquitectónicos, urbanísticos, sociales) en los centros históricos y los mecanismos de ejecución y financiación de proyectos.

En mayo del 2000 se logra inaugurar en Salvador de Bahía, Brasil, un nuevo tipo de seminarios internacionales. Por primera vez es condición previa que los actores locales de la ciudad anfitriona analicen la problemática particular de su ciudad y establezcan un diagnóstico. Luego, durante el seminario, todos los participantes (representantes de ciudades de América Latina y el Caribe, diversos especialistas en patrimonio y planificación urbana de la UNESCO, el BID y la UIA, arquitectos y representantes de organismos locales y europeos especializados en el tema) deben trabajar en grupos para elaborar respuestas a las necesidades expresadas por los actores locales en su diagnóstico. De esta forma se genera una situación dialéctica e interactiva entre expertos nacionales e internacionales. El programa Sirchal prefiere esta nueva dinámica a la participación pasiva en conferencias y coloquios, a las lecciones dadas por los expertos internacionales y a los modelos importados de otras latitudes.

En el caso de la revitalización del Pelourinho de Salvador de Bahía, el diagnóstico se recoge en el documento: «Recalificación, revitalización y durabilidad del centro histórico: un proyecto urbano», que sirve de base para los trabajos del seminario. Se identifican tres ejes de trabajo: el rol del sector público, la dimensión económica, social y política, así como la conservación integrada y los criterios de intervención.

En el mes de junio del 2000 se realiza en São Luis de Maranhão, Brasil, el primer $\mathrm{AEP}^{4}$. Esta nueva fase del programa Sirchal permite que un reducido grupo de expertos internacionales se concentre en un tema previamente definido en el diagnóstico preparado por los actores locales de la ciudad anfitriona. Es un tipo innovador de cooperación, cuyo objetivo es crear una 
dinámica sostenible a partir de soluciones inéditas, elaboradas conjuntamente por todos los actores locales y los expertos, que trabajan en estrecha colaboración con los organismos nacionales e internacionales de financiación.

El primer día del AEP los expertos locales e internacionales deben decidir, conjuntamente, qué hipótesis presentarán al día siguiente a las autoridades políticas, quienes tienen en sus manos la decisión respecto a la hipótesis que se desarrollará en el taller. En el caso de São Luis de Maranhão, el diagnóstico elaborado por los participantes ludovicenses se concentró en la vivienda en el centro histórico. La programación y la definición de los proyectos, así como la búsqueda y la integración de la financiación necesaria para realizar estudios y proyectos está en manos de un grupo de especialistas brasileños que continúa trabajando en colaboración con el programa Sirchal.

Los seminarios internacionales contribuyen a mantener viva la red, agilizando los intercambios y el flujo de información. Los talleres AEP no sólo permiten elaborar y definir programas y proyectos, sino que facilitan también el reconocimiento internacional del trabajo realizado por los actores locales. Además, posibilitan la concreción de los proyectos gracias a la participación de operadores locales o asociaciones entre operadores locales e internacionales.

Paralelamente se han desarrollado otras acciones, entre ellas la primera publicación conjunta del Ministerio de Cultura, la UNESCO y el BID, que incluye una evaluación crítica de las iniciativas tomadas en ese campo en las cinco últimas décadas, la publicación de un documento de trabajo, el «Glosario trilingüe Sirchal», que comprende conceptos utilizados en la revitalización de centros históricos y la difusión del libro Santiago Poniente: desarrollo urbano y patrimonio, realizado con el auspicio del programa Sirchal, los miembros de la red. Además, el Ministerio de Cultura y Comunicación de Francia y la Unión Internacional de Arquitectos han firmado un convenio para promover, en todos los países, el establecimiento del primer inventario del patrimonio arquitectónico del siglo XX. Cabe señalar que en el primer semestre del 2001 se realizará en Caracas, Venezuela, un taller cuyo objetivo es crear un espacio multimedia interactivo sobre la ciudad universitaria de Caracas, conjunto arquitectónico del siglo $\mathrm{xx}$ que ingresa el 30 de noviembre del 2000 en la lista del patrimonio mundial de la humanidad. Se puede afirmar que al poner de relieve la arquitectura del siglo Xx, se confirma la evolución en la noción de patrimonio.

La continuidad del programa Sirchal ha permitido, gracias a su sitio Internet (http://www.archi.fr/SIRCHAL) ${ }^{5}$, desarrollar sinergias con programas complementarios ${ }^{6}$ y sentar las bases de una red de redes que permitirá mantener una activa interacción a través de un motor de búsqueda.
Estos intercambios se plasman en el seguimiento de proyectos de vivienda en los centros históricos de Quito, Valparaíso y Santo Domingo, que cumplen cabalmente con uno de los principios sustentadores de Sirchal: la participación local en la financiación del proyecto. A partir de Salvador de Bahía y São Luis de Maranhão, la metodología utilizada tanto en los seminarios como en los talleres da un salto cualitativo. Aun cuando las experiencias de Ecuador, Chile y República Dominicana tienen dimensiones muy distintas, las tres reflejan el interés y la creciente participación del sector privado en la rehabilitación de viviendas en los centros históricos, sobre todo en República Dominicana, donde Pact Arim 93 (el operador de los tres proyectos) logra canalizar los instrumentos y la financiación del Banco Hipotecario Dominicano para realizar programas de vivienda en el centro histórico de Santo Domingo. En Brasil, la Caja Económica Federal anuncia, en el mes de diciembre del 2000 en São Luis de Maranhão, su decisión de reorientar parte de sus recursos financieros hacia un programa nacional destinado a la rehabilitación de vivienda en los centros históricos.

El proceso de revitalización de los centros históricos es determinante, ya que proporciona la forma e identidad necesarias para recrear y reestablecer la continuidad temporal, espacial y social de una ciudad. Esto no es cosa fácil, sobre todo cuando las condiciones económicas son precarias. La experiencia, sin embargo, demuestra que la búsqueda del desarrollo económico no debe obstaculizar el bienestar de los seres humanos.

En el futuro, es necesario reflexionar sobre nuevos conceptos de proyectos urbanos que correspondan a las realidades socioculturales de cada ciudad. El modelo de ciudad internacional no es válido y no existe una doctrina única que pueda utilizarse en todas las latitudes. Crear una ciudad a partir de criterios o necesidades particulares, ignorando los inevitables intercambios y los enriquecimientos mutuos, resulta imposible. Debemos, por lo tanto, construir ciudades adecuadas a nuestras culturas y a una utilización más humana y democrática de las mismas, sin olvidar que la participación de todos los actores es una condición sine qua non.

Tanto en Europa como en América Latina es necesario observar una actitud constructiva frente a estas mutaciones. La respuesta del programa Sirchal a la problemática que plantea la revitalización de los centros históricos queda demostrada en la evolución de las actividades organizadas desde la creación del programa, que a solicitud de los participantes han pasado de ser oportunidades de presentar - inter pares - proyectos y experiencias, a ser talleres dinámicos en los que se realiza un trabajo concreto y se utiliza el acervo de todos los participantes para aportar soluciones a los problemas identificados en el diagnóstico realizado por los actores locales. Asimismo, queda demostrado en el entusiasmo que manifiestan por este nuevo tipo de cooperación y desarrollo de proyectos sostenibles los res- 
ponsables de la revitalización de centros históricos, así como los demás asistentes a los seminarios y talleres y en la participación —cada vez más importante- de la sociedad civil y el sector privado en los programas de revitalización. Esta adecuación tam- bién se refleja en la creciente demanda proveniente de ciudades de América Latina y el Caribe para ser anfitrionas de un seminario o un taller: sin ir más lejos, este año se tiene planificado organizar seis talleres en distintas ciudades latinoamericanas.
* Arquitecto, coordinador del programa Sirchal para la DAPA (Dirección de Arquitectura y Patrimonio) del Ministerio de Cultura y Comunicación (MCC) y la AFAA (Asociación Francesa de Acción Artística) del Ministerio de Relaciones Exteriores (MAE), en el marco de un convenio existente entre ambos Ministerios. En colaboración con Monica Boyer, arquitecta, consultora del programa Sirchal.

${ }^{1}$ François Barré, director de Arquitectura y Patrimonio (DAPA) del Ministerio de Cultura y Comunicación de Francia de 1996 al 2000. Primer seminario Sirchal de revitalización de centros históricos en ciudades de América Latina y el Caribe. Maison de l'Amérique Latine, París, mayo de 1998.

${ }^{2}$ En Francia, por ejemplo, 71 millones de turistas, atraídos principalmente por la reputación del patrimonio, generaron en 1999 una balanza excedentaria de 70 mil millones de francos franceses.
${ }^{3}$ Convenio de cooperación sobre la revitalización y la valorización de centros históricos de América Latina y el Caribe entre el Ministerio de Cultura y Comunicación de Francia y el Banco Interamericano de Desarrollo, firmada el 10 de marzo de 1999 por Catherine Trautmann, ministra de Cultura de Francia, y Enrique Iglesias, Presidente del BID.

${ }^{4}$ Taller de expertise de proyectos, AEP por sus siglas en francés (atelier d'expertise des projets).

${ }^{5}$ A partir del sitio Internet del programa Sirchal se puede acceder a los sitios de todos los copartícipes del programa.

${ }^{6}$ Para empezar, con URB-AL, programa europeo de cooperación descentralizada entre ciudades latinoamericanas y europeas. 
